\title{
ALMOST SURE FUNCTIONAL LIMIT THEOREM FOR THE PRODUCT OF PARTIAL SUMS
}

\author{
KhurelbaAtar GonchigdanZan ${ }^{1}$
}

\begin{abstract}
We prove an almost sure functional limit theorem for the product of partial sums of i.i.d. positive random variables with finite second moment.
\end{abstract}

Mathematics Subject Classification. 60F05, 60F15.

Received March 25, 2008. Revised August 10, 2008.

\section{INTRODUCTION AND MAIN RESULT}

Limiting distributions of the product of partial sums of positive random variables have been widely studied in recent years. Arnold and Villaseñor [1] proved the limit theorem for the partial sum of a sequence of exponential random variables. Rempała and Wesołowski [4] proved it for any independent and identically distributed (i.i.d.) random variables with finite variance. Later, Qi [5] considered a sequence of random variables with $\alpha$-stable distribution and established the limit distribution of the product of the partial sums when $1<\alpha \leq 2$.

Recently, Zhang and Huang [6] proved the following invariance principle of the product of partial sums of i.i.d. positive random variables with mean $\mu>0$ and variance $\sigma^{2}$ :

$$
\left(\prod_{k=1}^{[n t]} \frac{S_{k}}{\mu k}\right)^{\frac{\mu}{\sigma \sqrt{n}}} \stackrel{\mathcal{D}}{\longrightarrow} \exp \left(\int_{0}^{t} \frac{W(s)}{s} \mathrm{~d} s\right) \text { as } n \rightarrow \infty .
$$

The goal of this paper is to obtain an almost sure version of the above invariance principle which can also be a functional version of the almost sure limit theorem obtained by Gonchigdanzan and Rempała [3]. Here is the result:

Theorem 1.1. Let $\left(X_{k}\right)_{k \geq 1}$ be a sequence of i.i.d. positive random variables with mean $\mu>0$ and variance $\sigma^{2}$ and let $S_{n}=X_{1}+\cdots+X_{n}$. Define a process $\left\{\xi_{n}(t): 0 \leq t \leq 1\right\}$ by

$$
\xi_{n}(t):=\left(\prod_{k=1}^{[n t]} \frac{S_{k}}{\mu k}\right)^{\frac{\mu}{\sigma \sqrt{n}}} .
$$

Keywords and phrases. Almost sure limit theorem, functional theorem, invariance principle, product of partial sums.

1 Department of Mathematical Sciences, University of Wisconsin, Stevens Point, WI 54481, USA; hurlee@uwsp.edu 
Let $F_{t}$ be the distribution function of the random variable on the right-hand side of (1.1). Then

$$
\frac{1}{\log n} \sum_{k=1}^{n} \frac{1}{k} \mathbf{I}\left(\xi_{k}(t) \leq x\right) \stackrel{\text { a.s. }}{\longrightarrow} F_{t}(x) \text { as } n \rightarrow \infty
$$

if and only if

$$
\frac{1}{\log n} \sum_{k=1}^{n} \frac{1}{k} \mathbf{P}\left(\xi_{k}(t) \leq x\right) \longrightarrow F_{t}(x) \text { as } n \rightarrow \infty
$$

Corollary 1.1. Let $\left(X_{k}\right)_{k \geq 1}$ be a sequence of i.i.d. positive random variables with mean $\mu>0$ and variance $\sigma^{2}$. Then we have

$$
\frac{1}{\log n} \sum_{k=1}^{n} \frac{1}{k} \mathbf{I}\left(\left(\prod_{j=1}^{[k t]} \frac{S_{j}}{\mu j}\right)^{\frac{\mu}{\sigma \sqrt{k}}} \leq x\right) \stackrel{\text { a.s. }}{\longrightarrow} F_{t}(x) \text { as } n \rightarrow \infty .
$$

\section{Auxiliary Results and proofs}

Throughout the paper $\log x$ and $\log \log x$ stand for $\ln (e \vee n)$ and $\ln \ln \left(n \vee e^{e}\right)$ respectively, and "«" is meant for the big "O" notation.

\subsection{Auxiliary results}

Lemma 2.1. Let $\left(Y_{n}\right)_{n \geq 1}$ be a sequence of random variables. Set $S_{n}=Y_{1}+\cdots+Y_{n}$. Then we have

$$
E\left(\max _{1 \leq k \leq n}\left|\sum_{j=1}^{k} \log \left(\frac{n+1}{j}\right) Y_{j}\right|\right) \leq 3 \log (n+1) E\left(\max _{1 \leq k \leq n}\left|S_{k}\right|\right) .
$$

Proof. Observe that

$$
\left|\sum_{j=1}^{k} \log \left(\frac{n+1}{j}\right) Y_{j}\right| \leq\left|\sum_{j=1}^{k} \log (n+1) Y_{j}\right|+\left|\sum_{j=2}^{k} \log j Y_{j}\right|=T_{1}+T_{2} .
$$

Obviously, $T_{1} \leq \log (n+1)\left|S_{k}\right|$ which implies

$$
\max _{1 \leq k \leq n} T_{1} \leq \log (n+1) \max _{1 \leq k \leq n}\left|S_{k}\right| .
$$

For the second term $T_{2}$ we have

$$
\begin{aligned}
\max _{2 \leq k \leq n} T_{2} & =\max _{2 \leq k \leq n}\left|\sum_{j=2}^{k} \log j Y_{j}\right|=\max _{2 \leq k \leq n}\left|\sum_{j=2}^{k}\left(Y_{j}+Y_{j+1}+\cdots+Y_{k}\right)(\log j-\log (j-1))\right| \\
& \leq \max _{2 \leq k \leq n} \sum_{j=2}^{k}\left|Y_{j}+Y_{j+1}+\cdots+Y_{k}\right|(\log j-\log (j-1)) \\
& \leq 2 \max _{2 \leq k \leq n}\left|Y_{1}+Y_{2}+\cdots+Y_{k}\right| \sum_{j=2}^{n}(\log j-\log (j-1)) \leq 2 \log (n+1) \max _{1 \leq k \leq n}\left|S_{k}\right| .
\end{aligned}
$$


Lemma 2.2. Let $\left(X_{k}\right)_{k \geq 1}$ be a sequence of i.i.d. positive random variables with mean $\mu$ and variance $\sigma^{2}$. Then setting $S_{n}=X_{1}+\cdots+X_{n}$ we have

$$
\max _{0 \leq t \leq 1}\left|\frac{\mu}{\sigma \sqrt{n}} \sum_{k=1}^{[n t]} \log \frac{S_{k}}{\mu k}-\frac{\mu}{\sigma \sqrt{n}} \sum_{k=1}^{[n t]}\left(\frac{S_{k}}{\mu k}-1\right)\right| \stackrel{\text { a.s. }}{\longrightarrow} 0 \text { as } n \rightarrow \infty .
$$

Proof. Note that $\log (x+1)=x-r(x)$ where $r(x) / x^{2} \rightarrow \frac{1}{2}$ as $x \rightarrow 0$. By the strong law of large numbers we have $S_{k} / k-\mu \stackrel{a . s .}{\longrightarrow} 0$ as $k \rightarrow \infty$.

Thus by the law of iterated logarithm we get

$$
\begin{aligned}
& \left|\frac{\mu}{\sigma \sqrt{n}} \sum_{k=1}^{[n t]} \log \frac{S_{k}}{\mu k}-\frac{\mu}{\sigma \sqrt{n}} \sum_{k=1}^{[n t]}\left(\frac{S_{k}}{\mu k}-1\right)\right| \stackrel{a . s .}{\ll} \frac{1}{\sigma \sqrt{n}} \sum_{k=1}^{[n t]}\left(\frac{S_{k}}{k}-\mu\right)^{2} \\
& \underset{\text { a.s. }}{\ll} \max _{0 \leq t \leq 1} \frac{1}{\sigma \sqrt{n}} \sum_{k=1}^{[n t]} \frac{1}{k} \log \log k \ll \frac{1}{\sigma \sqrt{n}} \log n \log \log n \rightarrow 0 .
\end{aligned}
$$

\subsection{Proof of Theorem $\mathbf{1 . 1}$}

We use Berkes and Dehling's [2] technique to prove our theorem. Observe that

$$
\sum_{k=1}^{n}\left(\frac{S_{k}}{k}-\mu\right)=\sum_{k=1}^{n} b_{k, n}\left(X_{k}-\mu\right)
$$

where $b_{k, n}=\sum_{j=k}^{n} 1 / j$. Hence by Lemma 2.2 it suffices to show that for any Borel-subset $A$ of $D[0,1]$

$$
\frac{1}{\log n} \sum_{k=2}^{n} \frac{1}{k} \mathbf{I}\left(\left(\frac{\hat{s}_{k}}{\sigma \sqrt{k}} \in A\right)-\mathbf{P}\left(\frac{\hat{s}_{k}}{\sigma \sqrt{k}} \in A\right)\right) \stackrel{\text { a.s. }}{\longrightarrow} 0 \text { as } n \rightarrow \infty
$$

where $\hat{s}_{n}=\sum_{i=1}^{[n t]} b_{i,[n t]}\left(X_{i}-\mu\right)$. From Berkes and Dehling [2] (p. 1647), to prove (2.1) it suffices to show that for any bounded Lipschitz function $f$ on $D[0,1]$ we have

$$
\frac{1}{\log n} \sum_{k=1}^{n} \frac{1}{k} \zeta_{k} \stackrel{a . s}{\longrightarrow} 0 \text { as } n \rightarrow \infty
$$

where $\zeta_{k}=f\left(\hat{s}_{k} / \sigma \sqrt{k}\right)-\mathbf{E} f\left(\hat{s}_{k} / \sigma \sqrt{k}\right)$. In fact, the following implies (2.2) (see p. 1648, Berkes and Dehling [2] for the proof):

$$
\mathbf{E}\left(\sum_{k=1}^{n} \frac{1}{k} \zeta_{k}\right)^{2} \ll \log ^{2} n(\log \log n)^{-\varepsilon} \text { for some } \varepsilon>0 .
$$

Therefore, showing (2.3) would be sufficient for the proof of Theorem 1.1. Observing

$$
\hat{s}_{l}-\hat{s}_{k}=b_{[k t]+1,[l t]}\left(S_{[k t]}-[k t] \mu\right)+\left(b_{[k t]+1,[l t]}\left(X_{[k t]+1}-\mu\right)+\cdots+b_{[l t],[l t]}\left(X_{[l t]}-\mu\right)\right)
$$

for $l \geq k$ we find that $\hat{s}_{l}-\hat{s}_{k}-b_{[k t]+1,[l t]}\left(S_{[k t]}-\mu[k t]\right)$ is independent of $\hat{s}_{[k t]}$ which implies that

$$
\operatorname{Cov}\left(f\left(\frac{\hat{s}_{k}}{\sigma \sqrt{k}}\right), f\left(\frac{\hat{s}_{l}-\hat{s}_{k}-b_{[k t]+1,[l t]}\left(S_{[k t]}-\mu[k t]\right)}{\sigma \sqrt{l}}\right)\right)=0 \text { for } l \geq k
$$


By the Lipschitz property of $f$

$$
\begin{aligned}
\left|\mathbf{E}\left(\zeta_{k} \zeta_{l}\right)\right| & \ll \mid \operatorname{Cov}\left(f\left(\frac{\hat{s}_{k}}{\sigma \sqrt{k}}\right), f\left(\frac{\hat{s}_{l}}{\sigma \sqrt{l}}\right)-f\left(\frac{\left|\hat{s}_{l}-\hat{s}_{k}-b_{[k t]+1,[l t]}\left(S_{[k t]}-\mu[k t]\right)\right|}{\sigma \sqrt{l}}\right) \mid\right. \\
& \ll \mathbf{E}\left(\max _{0 \leq t \leq 1} \frac{\left|\hat{s}_{k}+b_{[k t]+1,[l t]}\left(S_{[k t]}-\mu[k t]\right)\right|}{\sigma \sqrt{l}}\right) \\
& \ll \mathbf{E}\left(\max _{0 \leq t \leq 1} \frac{\left|\hat{s}_{k}\right|}{\sigma \sqrt{l}}\right)+\mathbf{E}\left(\max _{0 \leq t \leq 1} \frac{\left|b_{[k t]+1,[l t]}\left(S_{[k t]}-\mu[k t]\right)\right|}{\sigma \sqrt{l}}\right) \\
& \ll\left(\frac{k}{l}\right)^{1 / 2} \mathbf{E}\left(\max _{0 \leq t \leq 1} \frac{\left|\hat{s}_{k}\right|}{\sigma \sqrt{k}}\right)+\left(\frac{k}{l}\right)^{1 / 2} \mathbf{E}\left(\max _{0 \leq t \leq 1} b_{[k t]+1,[l t]} \frac{\left|S_{[k t]}-\mu[k t]\right|}{\sigma \sqrt{k}}\right) .
\end{aligned}
$$

Since $\max _{0 \leq t \leq 1} b_{[k t]+1,[l t]}=\log (l / k) \ll(l / k)^{\gamma}$ (we choose $\left.0<\gamma<1 / 2\right)$

$$
\begin{aligned}
\left|\mathbf{E}\left(\zeta_{k} \zeta_{l}\right)\right| & \ll\left(\frac{k}{l}\right)^{1 / 2} \mathbf{E}\left(\max _{0 \leq t \leq 1} \frac{1}{\sigma \sqrt{k}}\left|\sum_{i=1}^{[k t]} b_{i, k}\left(X_{i}-\mu\right)\right|\right)+\left(\frac{k}{l}\right)^{1 / 2-\gamma} \mathbf{E}\left(\max _{0 \leq t \leq 1} \frac{\left|S_{[k t]}-\mu[k t]\right|}{\sigma \sqrt{k}}\right) \\
& =\left(\frac{k}{l}\right)^{1 / 2} \mathbf{E}\left(\max _{0 \leq j \leq k} \frac{1}{\sigma \sqrt{k}}\left|\sum_{i=1}^{j} b_{i, k}\left(X_{i}-\mu\right)\right|\right)+\left(\frac{k}{l}\right)^{1 / 2-\gamma} \mathbf{E}\left(\max _{0 \leq j \leq k} \frac{\left|S_{j}-\mu j\right|}{\sigma \sqrt{k}}\right) \\
& =M_{1}+M_{2} .
\end{aligned}
$$

Now applying Lemma 2.1 to $M_{1}$ we obtain

$$
\begin{aligned}
\left|\mathbf{E}\left(\zeta_{k} \zeta_{l}\right)\right| & \ll\left(\frac{k}{l}\right)^{1 / 2} \log k \mathbf{E}\left(\max _{1 \leq j \leq k} \frac{1}{\sigma \sqrt{k}}\left|\sum_{i=1}^{j}\left(X_{i}-\mu\right)\right|\right)+\left(\frac{k}{l}\right)^{1 / 2-\gamma} \mathbf{E}\left(\max _{1 \leq j \leq k} \frac{\left|S_{j}-\mu j\right|}{\sigma \sqrt{k}}\right) \\
& \ll\left|\mathbf{E}\left(\zeta_{k} \zeta_{l}\right)\right| \ll \log k\left(\frac{k}{l}\right)^{1 / 2-\gamma} \mathbf{E}\left(\max _{1 \leq j \leq k} \frac{\left|S_{j}-\mu j\right|}{\sigma \sqrt{k}}\right) \ll \log k\left(\frac{k}{l}\right)^{\gamma^{\prime}}
\end{aligned}
$$

where $0<\gamma^{\prime}<1 / 2-\gamma$.

On the other hand $\mathbf{E}\left(\zeta_{k} \zeta_{l}\right) \ll 1$ because $\zeta_{k}$ is bounded. Hence we have the following estimate for $\mathbf{E}\left(\zeta_{k} \zeta_{l}\right)$ :

$$
\mathbf{E}\left(\zeta_{k} \zeta_{l}\right) \ll \begin{cases}1, & \text { if } l / k \leq \exp \left((\log n)^{1-\varepsilon}\right) \\ (k / l)^{\gamma^{\prime}} \log k, & \text { if } l / k \geq \exp \left((\log n)^{1-\varepsilon}\right)\end{cases}
$$

where $\varepsilon$ is any positive number. Hence,

$$
\sum_{\substack{1 \leq k \leq l \leq n \\ l / k \leq \exp (\log n)^{1-\varepsilon}}} \frac{\mathbf{E}\left(\zeta_{k} \zeta_{l}\right)}{k l} \leq \sum_{1 \leq k \leq n} \frac{1}{k} \sum_{k \leq l \leq k e^{(\log n)^{1-\varepsilon}}} \frac{1}{l} \ll \sum_{k=1}^{n} \frac{1}{k} \log ^{1-\varepsilon} n \ll \log ^{2-\varepsilon} n
$$

and

$$
\sum_{\substack{1 \leq l \leq k \leq n \\ l / k \geq \exp (\log n)^{1-\varepsilon}}} \frac{\mathbf{E}\left(\zeta_{k} \zeta_{l}\right)}{k l} \leq \mathrm{e}^{-\gamma^{\prime}(\log n)^{1-\varepsilon}} \log n \sum_{1 \leq k \leq l \leq n} \frac{1}{k l} \ll \mathrm{e}^{-\gamma^{\prime}(\log n)^{1-\varepsilon}} \log ^{3} n \ll \log ^{2-\varepsilon} n .
$$

Thus (2.4) and (2.5) immediately imply (2.3) which completes the proof of Theorem 1.1. 
Acknowledgements. I am very thankful to the referees for their insightful comments. I would also like to express my gratitude to Prof. C. Endre for his encouragement.

\section{REFERENCES}

[1] B.C. Arnold and J.A. Villaseñor, The asymptotic distribution of sums of records. Extremes 1 (1998) 351-363.

[2] I. Berkes and H. Dehling, Some limit theorems in log density. Ann. Probab. 21 (1993) 1640-1670.

[3] K. Gonchigdanzan and G. Rempała, A note on the almost sure limit theorem for the product of partial sums. Appl. Math. Lett. 19 (2006) 191-196.

[4] G. Rempała and J. Wesołowski, Asymptotics for products of sums and U-statistics. Electron. Commun. Probab. 7 (2002) $47-54$.

[5] Y. Qi, Limit distributions for products of sums. Statist. Probab. Lett. 62 (2003) 93-100.

[6] L. Zhang and W. Huang, A note on the invariance principle of the product of sums of random variables. Electron. Commun. Probab. 12 (2007) 51-56. 